\title{
New Interplanetary Scintillation Array in China for Space Weather
}

\author{
Yihua Yan ${ }^{1,2}$, Wei Wang ${ }^{1}$, Linjie Chen ${ }^{1}$, Fei Liu ${ }^{1}$, Lihong Geng ${ }^{1}$, Zhijun Chen ${ }^{1}$ \\ ${ }^{1}$ CAS KLSA, NAOC, Beijing 100101, China \\ ${ }^{2}$ School of Astronomy and Space Sciences, University of CAS, Beijing 100049, China
}

\author{
E mail (yyh@nao.cas.cn)
}

Accepted: 19 November 2018

\begin{abstract}
Interplanetary Scintillation (IPS) is a useful ground-based method to investigate solar wind structure and its parameters through the scintillation of distant, compact radio sources at radio wavelengths. Current worldwide IPS facilities include both single-site systems (which are typically a large telescope or array), and multi-site systems (which are typically more varied telescope/array sizes). To combine the advantages of both of these system types, we propose a new design for the IPS telescope in China, implementing a large collecting area at one main site, with smaller collecting areas at the other sites. This new IPS telescope concept is a part of the Phase-II Meridian Space Weather Monitoring Project under the 2016-2020 National Infrastructure Program in China to be constructed in near future. Some specifications and basic considerations of this telescope are described in this paper.
\end{abstract}

(c) 2018 BBSCS RN SWS. All rights reserved

Keywords: : IPS, array, radio telescope, space weather

\section{INTRODUCTION}

There are two ways to investigate the basic properties of the solar wind plasma in the inner heliosphere: (i) through direct insitu measurements, or (ii) via indirect remote-sensing observations (e.g., Tokumaru 2013). Compared to in situ measurements, which typically require expensive missions, ground-based observations are usually more economically effective. The interplanetary scintillation (IPS) of compact radio sources at meter to centimeter wavelengths is an effective tool to investigate the structures and properties of the solar wind throughout the inner heliosphere. Observations of IPS, usually at a frequency of $327 \mathrm{MHz}$, have been carried out since 1970s in the world. Observations of IPS are extremely useful in determining the physical parameters and structure of the solar wind throughout the inner heliosphere (e.g., Manoharan 1993, Kojima et al. 1995, Breen et al. 2002, Manoharan \& Agalya 2011, Bisi et al. 2007, 2010, Chang et al. 2016, and Morgan et al. 2018).

It is desirable to achieve high sensitivity of the telescope for observations of IPS. Therefore, a sufficiently-large collecting area is necessary for an IPS-capable telescope. There are different technical solutions to carry out observations of IPS: a traditional steerable parabolic radio telescope, a very large cylinder antenna, or an array type system made up of many small antennas/receivers. At present, the main IPS dedicated telescopes are the Institute for Space-Earth Environmental Research (ISEE), Nagoya University stations in Japan, the Ooty radio telescope (ORT) in India, and the Mexican Array Radio Telescope (MEXART) in Mexico. Many other radio-telescope systems are used mainly on a 'campaign' basis.

The ISEE IPS telescopes consist of four cylinder antennas with reflector size $88 \mathrm{~m} * 38 \mathrm{~m}, 20 \mathrm{~m} * 100 \mathrm{~m}, 20 \mathrm{~m} * 100 \mathrm{~m}$, and $27 \mathrm{~m} * 73.5 \mathrm{~m}$. After the $1990 \mathrm{~s}$, the observations were improved with innovative methods for data analysis (e.g. Tokumaru 2013). Even though the number of radio sources used for ISEE observations of IPS are fewer than that for the ORT (below), it has been very successful to study the three-dimensional (3-D) structure of CMEs and their formation processes (e.g. Tokumaru et al. 2000, 2003, 2005, 2006, 2007). However, it is expected that the 3-D solar wind structure would be better modeled and reconstructed with more radio sources employed (e.g. Jackson et al. 2010, 2011, 2013).

The ORT is an offset parabolic cylinder, $530 \mathrm{~m} \times 30 \mathrm{~m}$ in size which was commissioned in 1970 (Swarup et al. 1971). ORT has been recently upgraded to the Ooty Wide Field Array (OWFA), which has both a larger instantaneous bandwidth as well as a significantly-larger field of view (Subrahmanya et al., 2017). Its sensitivity is upgraded to $8 \mathrm{mJy}$ with 1 second integration time, and more than 10,000 radio sources can be observed by the OWFA. In the distribution of radio sources, more than $80 \%$ of them can be used as IPS sources (i.e., they exhibit scintillation because they have one or more compact components). These sources are valuable to study solar-wind transients (e.g., Coronal Mass Ejections-CMEs and interplanetary Co-rotating Interaction Regions-CIRs) as well as the ambient solar wind.

The MEXART consists of a $64 \times 64$ (4096) full-wavelength dipole antenna array, operating at $140 \mathrm{MHz}$, with a bandwidth of 2 MHz, occupying about 9,660 square meters $(69 \mathrm{~m} \times 140 \mathrm{~m})($ e.g., Mejia-Ambriz 2010). Only some strong IPS radio sources can be detected at present because the sensitivity is currently limited.

Miyun Synthesis Radio Telescope (MSRT), located at the Miyun observatory in Beijing, China was used in the phased array mode at $232 \mathrm{MHz}$ for IPS studies in the 1990s (Wu et al. 2001). Later, an IPS-observation system using the $50 \mathrm{~m}$ parabolic radio telescope, used S/X and UHF bands for IPS experiments in China (Liu et al. 2010)

Compared with single-site observations, the flow velocities of the solar wind can be derived from simultaneous observations of IPS at multi-stations (e.g. Kakinuma et al. 1973). The projected solar wind speed and other parameters can be determined directly by cross-correlations of the multi-site data, whereas for singlestation observations of IPS, these parameters have to be deduced from proper models of the solar wind. In order to derive 3-D solar wind structures, it is very important and necessary to have large collecting area so as to observe more radio sources. For observations of IPS with more than one frequency, one can achieve higher accuracy in the calculation of the characteristic frequency, reduce the effects from the variation of the solar wind parameters, and obtain higher sensitivity (Scott et al. 1983, Zhang 2007).

A well-used IPS space-weather technique actually applies Computer Assisted Tomography to reconstruct the 3-D solar wind structure (e.g., Jackson et al. 2010, 2011, 2013, Bisi 2016, and references therein). It would benefit from receiving direct results from observations of a larger number of radio sources. Therefore, a new IPS telescope solution is proposed which includes one main site and two sub-sites. At the main site there are three cylinder antennas placed side by side each having a large size of $140 \mathrm{~m}$ in $\mathrm{N}-\mathrm{S}$ direction and $40 \mathrm{~m}$ in E-W direction, plus an available $20 \mathrm{~m}$ parabolic antenna (dish 1) used for the calibration of a present solar radio array at the main site. At the two sub-sites, there is a 16 m parabolic antenna (dish 2) with cryogenically cooled receiver at 
each site. In this way, we can achieve observing more radio sources with direct measurements for strong sources so that the 3D solar-wind structure can be better reconstructed than with the currently-available real-time facilities alone (this is predominantly ISEE only at present). The detailed description of the new IPS concept is discussed in the next section.

\section{DESCRIPTION OF NEW IPS TELESCOPE ARRAY}

\subsection{Arrangement of IPS Telescope Array System}

As mentioned in the previous section, we are aiming to utilize the advantages of the multi-site and dual-frequency observations of IPS, so we propose a new telescope design for observations of IPS while under a limited budget. The whole IPS telescope array is made up of three sites: one main site with the largest collecting area, and two sub-sites each with an intermediate-size collecting area. At each site the antennas not only observe IPS sources independently with dual frequencies (and the dish antennas observe one more frequency than the cylinder antenna), but they also work together in multi-site mode as a whole.

In order to scan the sky quickly and for other scientific targets, the cylinder antennas are designed to be rotatable in the E-W direction, but do not allow for tracking of sources on the sky because of the limited budget. Therefore, they can be set to point to a direction in short time and wait for the passing of the IPS sources. Each cylinder antenna has a line focus, along which an array of 304 dipoles is placed. Each feed has two dipoles, which can work at dual frequencies, $327 \mathrm{MHz}$ and $654 \mathrm{MHz}$. Each dipole is connected by a Low Noise Amplifier (LNA). The output of each LNA is digitalized with an AD converter. The signals are bandpass sampled to give an effective bandwidth of $\sim 40 \mathrm{MHz}$. Then the digital signal is transmitted through optical fiber to the control room. After some amplifiers and filters, the signals from each dipole can be easily combined to form multiple-beams in the digital beam-forming unit and without the need for a complicated phase-shifter arrangement. In total, $36(3 \times 12)$ beams from the cylinders will be simultaneously formed. The efficiency of antenna aperture is about $65 \sim 70 \%$. The system temperature is about $120 \mathrm{~K}$ with a well-designed LNA at these two frequencies. The sensitivity of the main station is about $4.4 \mathrm{mJy}$ with a onesecond integration time. The sensitivity is better than the performance of Ooty IPS telescope. For each IPS source, the observing time is determined by the width of main beam at half power of the telescope (since the source can be tracked in E-W direction with the synthesis beam) depending on beamforming technology, i.e., about 2.5 minutes for $654 \mathrm{MHz}$ and 5 minutes for $327 \mathrm{MHz}$. The actual data sampling cadence could be as fast as tens ms.

At each of the two sub-sites, there is a $\sim 16 \mathrm{~m}$ parabolic dish antenna with cryogenically cooled receiver. The antenna is azimuth-elevation mounted. Due to the size of each feed, a rotated feed support structure is considered. One side of this structure is for the $327 \mathrm{MHz}$ and $654 \mathrm{MHz}$ feeds, and the other side is for a $1,420 \mathrm{MHz}$ feed. The system temperature is about $120 \mathrm{~K}$. Therefore, compared to room temperature, the sensitivity of the $16 \mathrm{~m}$ dish can be greatly improved. Some relatively-strong radio sources can be observed. The RF signals at three frequencies (327 $\mathrm{MHz}, 654 \mathrm{MHz}$ and 1,420 MHz) from IPS sources are transmitted to the indoor unit by optical fibers. The indoor equipment includes optic receivers, analogous receivers, AD converters, digital correlation receivers, local oscillators, monitors, computers, and so on. After the $\mathrm{AD}$ converter, the digitized data will be transmitted to a data computer server in the observing room. These observations can be correlated with the data observed by the cylinder and the $20 \mathrm{~m}$ dish telescopes in main station.

\subsection{Specifications and Telescope Configuration}

The main specifications are listed in Table 1 and the configuration of the IPS Telescope Array is shown in Figure 1.

Sub Site: Abaga

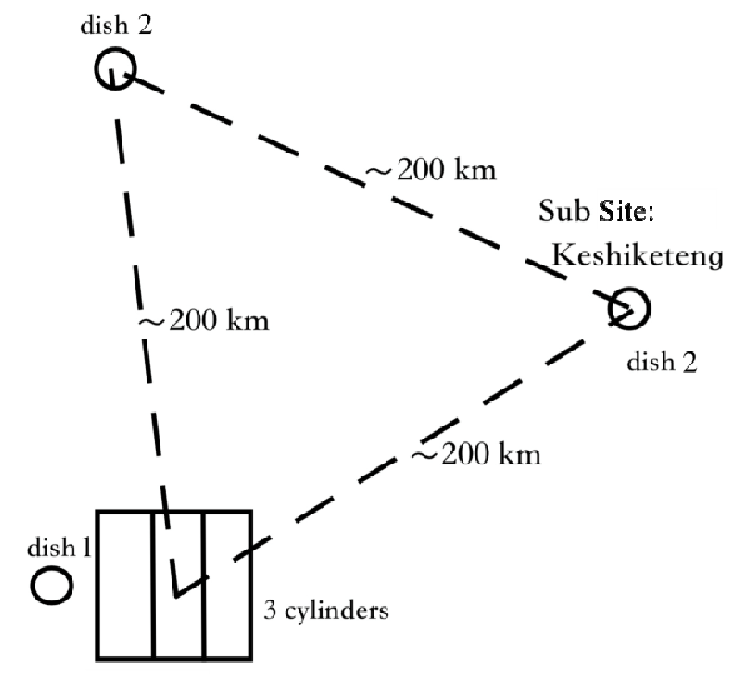

Main Site: Mingantu

Figure 1. Configuration of the IPS Telescope Array

\subsection{Data Transmission and Processing}

The data center for storage, processing, and distribution of the data is located at the main site. The data transmission among the three stations depends on renting commercial optical cable. A computer cluster environment is proposed for the IPS data processing. The system should be realized efficiently with the load balance, high compatibility, and easy-upgrading capacity. An acquisition server will be used to receive observational data from the digital receiver and store the raw IPS data to the storage system. Meanwhile, the acquisition server would repeatedly forward the observational data to monitoring servers for real-time monitoring. The data from the main site and both smaller sites will be processed using a high-performance distributed data-processing pipeline.

\section{DISCUSSION}

The design of a new multi-site IPS telescope is described briefly. The cylinder antennas at the main site are rotatable, but will not have tracking capability, and will have a total collecting area slightly larger than the ORT. Therefore, more radio sources should be able to be observed provided there is no loss in sensitivity. It is hoped that the additional data for use in 3-D tomography and models will mean that the solar-wind structure can be better reconstructed than from the currently-available facilities alone.

\section{SUMMARY}

In summary, a new IPS telescope concept has been proposed with the following features: large collecting areas with high sensitivity, multiple sites to measure solar-wind parameters directly for strong sources, and dual-frequencies to achieve higher accuracy in the calculation of the characteristic frequency. This new IPS telescope concept is a part of the Phase-II Meridian Space Weather Monitoring Project under the 2016-2020 National Infrastructure Program in China to be constructed in near future. Therefore, it will play an important role for space weather studies when finished. As the Parker Solar Probe and Solar Orbiter will be approaching the Sun, then the combination of in-situ measurements and remote-sensing observations will significantly improve our understanding of solar wind properties and of spaceweather processes. 
Table 1. Main Specifications

\begin{tabular}{|c|c|}
\hline \multirow{2}{*}{ Antenna } & Cylinder: $3 \times 140 \mathrm{~m} \times 40 \mathrm{~m}$ \\
\hline & Dish $1 \&$ Dish 2: $1 \times \varnothing 20 \mathrm{~m}$ and $2 \times \varnothing 16 \mathrm{~m}$ \\
\hline Frequency & $327 \mathrm{MHz}, 654 \mathrm{MHz}, 1420 \mathrm{MHz}\left(^{*}\right)$ \\
\hline Frequency Bandwidth & $40 \mathrm{MHz}$ \\
\hline Frequency Resolution & $39 \mathrm{KHz}$ \\
\hline \multirow{2}{*}{$\begin{array}{l}\text { Beam } \\
\text { (with } 36=3 \times 12 \text { simultaneously-formed beams for } \\
\text { the cylinder antennas) }\end{array}$} & $\begin{array}{c}\text { Main site Cylinder: 1.60× 27' @ } 327 \mathrm{MHz} \\
0.8^{\circ} \times 13.7^{\prime} @ 654 \mathrm{MHz} \\
\text { Dish 1: } 0.74^{\circ} \times 0.74^{\circ} @ 1420 \mathrm{MHz}\end{array}$ \\
\hline & $\begin{array}{c}\text { Sub-sites Dish 2: } 4^{\circ} @ 327 \mathrm{MHz} \\
2^{\circ} @ 654 \mathrm{MHz} \\
0.9^{\circ} @ 1420 \mathrm{MHz}\end{array}$ \\
\hline \multirow{2}{*}{ System Temperature } & Cylinder: $\sim 110 \mathrm{~K}$ \\
\hline & Dish 1 \& 2: $\quad \sim 120 \mathrm{~K}$ \\
\hline System Gain & $\sim 80 \mathrm{~dB}$ \\
\hline ADC & 12 bits@ 100 MSPS \\
\hline \multirow[t]{2}{*}{ Sensitivity } & $\begin{array}{l}\text { Main-site: } \\
\text { Cylinder: } 4.4 \text { mJy@1s with 65\% efficiency } \\
\text { Dish 1: 167 mJy@1s with 70\% efficiency }\end{array}$ \\
\hline & Sub-sites: 261 mJy@1s with 70\% efficiency \\
\hline
\end{tabular}

(* only available for the 20-m and 16-m dish antennas.)

\section{ACKNOWLEDGEMENTS}

Profs. Ramesh and Manoharan are gratefully acknowledged for comments and suggestions to improve the design for this IPS telescope. We also like to thank Profs. Xueshang Feng, Ming Xiong, Jiansen He, and Dr. Lijia Liu for helpful discussion on this new IPS concept. The work is supported by National Natural Science Foundation of China grants 11433006, 11790301,11773043, and the Meridian-II Project under National Infrastructure Program in China.

\section{REFERENCES}

Bisi, M. M., Fallows, R. A., Breen, A. R., Habbal, S. Rifai, Jones, R. A.: 2007, J. Geophys. Res.: Space Physics, 112, A06101.

Bisi, M. M., Jackson, B. V., Breen, A. R., Dorrian, G. D., Fallows, R . A., Clover, J.M., Hick, P.P.: 2010, Solar Phys., 265, 233

Bisi, M.: 2016, Heliophysics with radio scintillation and occultation. In C. Schrijver, F. Bagenal, \& J. Sojka (Eds.), Heliophysics: Active Stars, their Astrospheres, and Impacts on Planetary Environments (pp. 289-326). Cambridge: Cambridge University Press. doi:10.1017/CBO9781316106778.014

Breen, A. R., Thomasson, P., Jordan, C. A., Tappin, S. J., Fallows, R. A ., Canals, A., Moran, P. J.: 2002, Adv. Space Res., 30, 433.

Chang, Oyuki, Gonzalez-Esparza, J. A., Mejia-Ambriz, J.: 2016, Adv. Space Res., 57, 1307.

Jackson, B. V., Hick, P. P., Bisi, M. M., Clover, J. M., Buffington, A.: 2010, Solar Physics, 265, 245.

Jackson, B. V., Hick, P. P., Buffington, A., Bisi, M. M., Clover, J. M., Tokumaru, M., Kojima, M., Fujiki, K.: 2011, J. Atmos. Solar-Terrestrial Phys., 73, 1214.
Jackson, B. V., Clover, J. M., Hick, P. P., Buffington, A., Bisi, M. M., Tokumaru, M.:2013, Solar Physics, 285, 151.

Kakinuma, T., Washimi, H, Kojima, M.: 1973, PASJ, 25, 271.

Kojima M., Asai K., Kozuka Y., Misawa H., Watanabe H., Yamauchi Y.: 1995, Adv. Space Res., 16, 110.

Liu, L.J., Zhang, X.Z., Li, J. B., Manoharan, P. K., Liu, Z. Y., Peng, B.: 2010, Res. Astron. Astrophys. 10, 577.

Manoharan, P. K.: 1993, Solar Phys. 148, 153.

Manoharan, P. K. and Agalya, G.: 2011, Adv. Geosciences, 27, 165.

Mejia-Ambriz, J.C., Villanueva-Hernandez, P., Gonzalez-Esparza, J.A., Aguilar-Rodriguez E., Jeyakumar, S.: 2010, Solar Phys. 265, 309.

Morgan, J. S., Macquart, J.-P., Ekers, R., Chhetri, R., Tokumaru, M., Manoharan, P. K., Tremblay, S., Bisi, M. M., Jackson, B. V.: 2018, MNRAS, 473, 2965.

Scott, S. L., Rickett, B. J., Armstrong, J. W.: 1983, Astron. Astrophys., 123, 191-206.

Swarup, G., Kapahi, V. K., Velusamy, T., Ananthakrishnan, S., Balasubramanian, V., Gopal-Krishna, Pramesh-Rao, A., Subrahmanya, C. R., Kulkarni, V. K.: 1971, Current Science, 60. 